\title{
Control of SRF binding to CArG box chromatin regulates smooth muscle gene expression in vivo
}

\author{
Oliver G. McDonald, Brian R. Wamhoff, Mark H. Hoofnagle, and Gary K. Owens
}

Department of Molecular Physiology and Biological Physics, University of Virginia Health Sciences Center, Charlottesville, Virginia, USA.

\begin{abstract}
Precise control of SMC transcription plays a major role in vascular development and pathophysiology. Serum response factor (SRF) controls SMC gene transcription via binding to CArG box DNA sequences found within genes that exhibit SMC-restricted expression. However, the mechanisms that regulate SRF association with CArG box DNA within native chromatin of these genes are unknown. Here we report that SMC-restricted binding of SRF to murine SMC gene CArG box chromatin is associated with patterns of posttranslational histone modifications within this chromatin that are specific to the SMC lineage in culture and in vivo, including methylation and acetylation to histone $\mathrm{H} 3$ and $\mathrm{H} 4$ residues. We found that the promyogenic SRF coactivator myocardin increased SRF association with methylated histones and CArG box chromatin during activation of SMC gene expression. In contrast, the myogenic repressor Kruppel-like factor 4 recruited histone $\mathrm{H} 4$ deacetylase activity to SMC genes and blocked SRF association with methylated histones and CArG box chromatin during repression of SMC gene expression. Finally, we observed deacetylation of histone $\mathrm{H} 4$ coupled with loss of SRF binding during suppression of SMC differentiation in response to vascular injury. Taken together, these findings provide novel evidence that SMC-selective epigenetic control of SRF binding to chromatin plays a key role in regulation of SMC gene expression in response to pathophysiological stimuli in vivo.
\end{abstract}

\section{Introduction}

The differentiated state of SMCs plays a key role in the pathogenesis of several major human diseases, including atherosclerosis, SMC restenosis after vascular injury, hypertension, asthma, reproductive disorders, and cancer (1). Unlike terminally differentiated cells, SMCs can switch between differentiated and dedifferentiated phenotypes in response to changes in local environmental cues (a process referred to as SMC phenotypic switching or phenotypic modulation). For example, in response to vascular injury, differentiated SMCs downregulate expression of SMC-specific contractile genes, proliferate, migrate into the vessel intima, and synthesize collagens and matrix metalloproteinases (1). This process is critical for repair of vascular injury, but if dysregulated can result in restenosis after percutaneous vascular interventions such as stenting or angioplasty. There is evidence that SMCs or smooth muscle-like cells within vascular lesions can be derived from a number of sources (2), including preexisting SMCs from the vessel media (1), adventitial smooth muscle progenitor cells (3), and circulating bone marrow-derived stem cells $(4,5)$. Whatever their origin, a hallmark of this SMC phenotypic plasticity is transcriptional repression of SMC-specific differentiation genes, such as $\alpha$-SMA, smooth muscle-myosin heavy chain (SM-MHC), and SM22 $\alpha$ (6). Therefore, a key challenge in the field has been to determine the molecular

Nonstandard abbreviations used: ChIP, chromatin immunoprecipitation; EMSA, electrophoretic mobility shift assay; H4Ac, H4 acetylation; HAT, histone acetyltransferase; HDAC, histone deacetylase; H3K4dMe, H3 Lys4 di-methylation; HMT, histone methyltransferase; KLF4, Kruppel-like factor 4; SM-MHC, smooth muscle-myosin beavy chain; SRF, serum response factor; TSA, trichostatin A.

Conflict of interest: The authors have declared that no conflict of interest exists.

Citation for this article: J. Clin. Invest. 116:36-48 (2006).

doi:10.1172/JCI26505. mechanisms whereby SMC genes are transcriptionally regulated under physiological and pathophysiological conditions.

Evidence from our laboratory and others has firmly established that CArG box $\left[\mathrm{CC}(\mathrm{A} / \mathrm{T})_{6} \mathrm{GG}\right]$ DNA sequences present within the promoters of SMC genes play a pivotal role in controlling their transcription (7). CArG boxes serve as binding sites for serum response factor (SRF), a ubiquitously expressed MADS box transcription factor that binds as a homodimer to CArG box DNA to activate transcription of genes involved in both muscle differentiation and proliferation (e.g., c-fos) (7).The SRF-CArG association is required for transcriptional activation of SMC genes (7), and this association can also mediate transcriptional repression of these genes in response to pathological environmental cues in culture and in vivo (8-10). Thus, the SRF-CArG interaction is a critical convergence point for signals that either activate SMC gene expression to promote SMC differentiation under physiological environments or repress SMC gene expression during pathophysiological conditions. Because of this, control of SRF binding to the CArG boxes of these genes likely represents a major mechanism for regulation of SMC gene expression. However, the molecular mechanisms that control this process are poorly defined.

Recent studies by our laboratory and others have revealed that regulation of SMC gene expression is dependent, at least in part, on the ability of SRF to bind CArG box DNA sequences within the context of intact chromatin (10-15). This is important because it is well recognized that chromatin structure is a critical determinant of how permissive DNA sequences are to transcription factor binding (16). Thus, an understanding of the chromatin structure of SMC gene promoters could provide insight into how SRF regulates SMC gene expression.

At its most fundamental level, chromatin is organized into repeating units of nucleosomes, the basic building blocks of chro- 
A

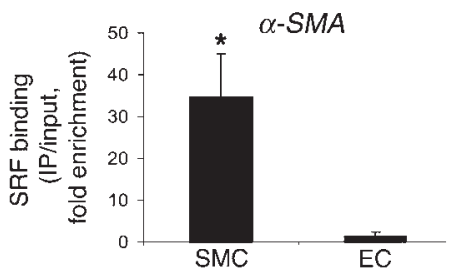

B

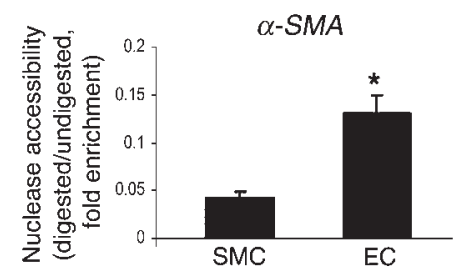

C

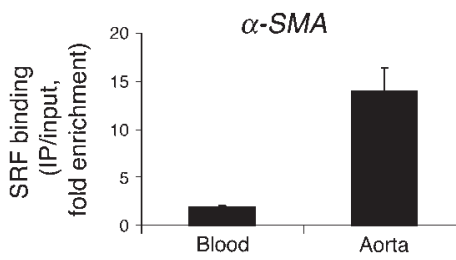

D
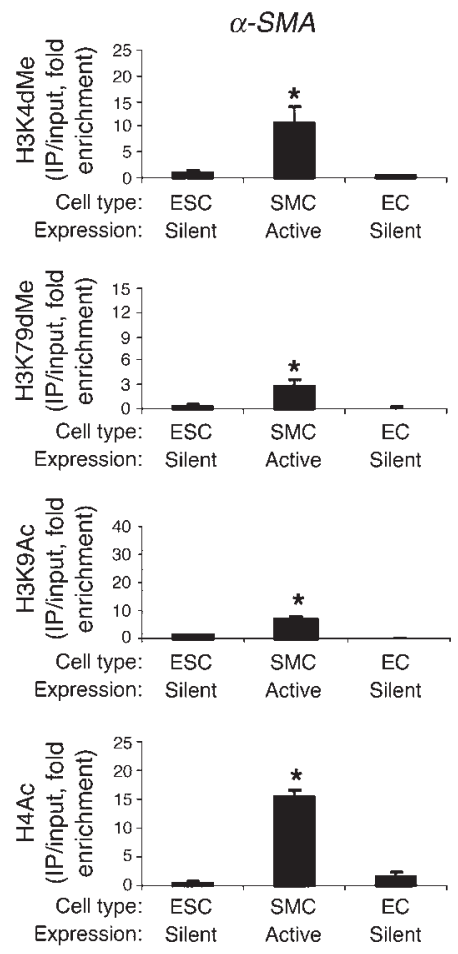
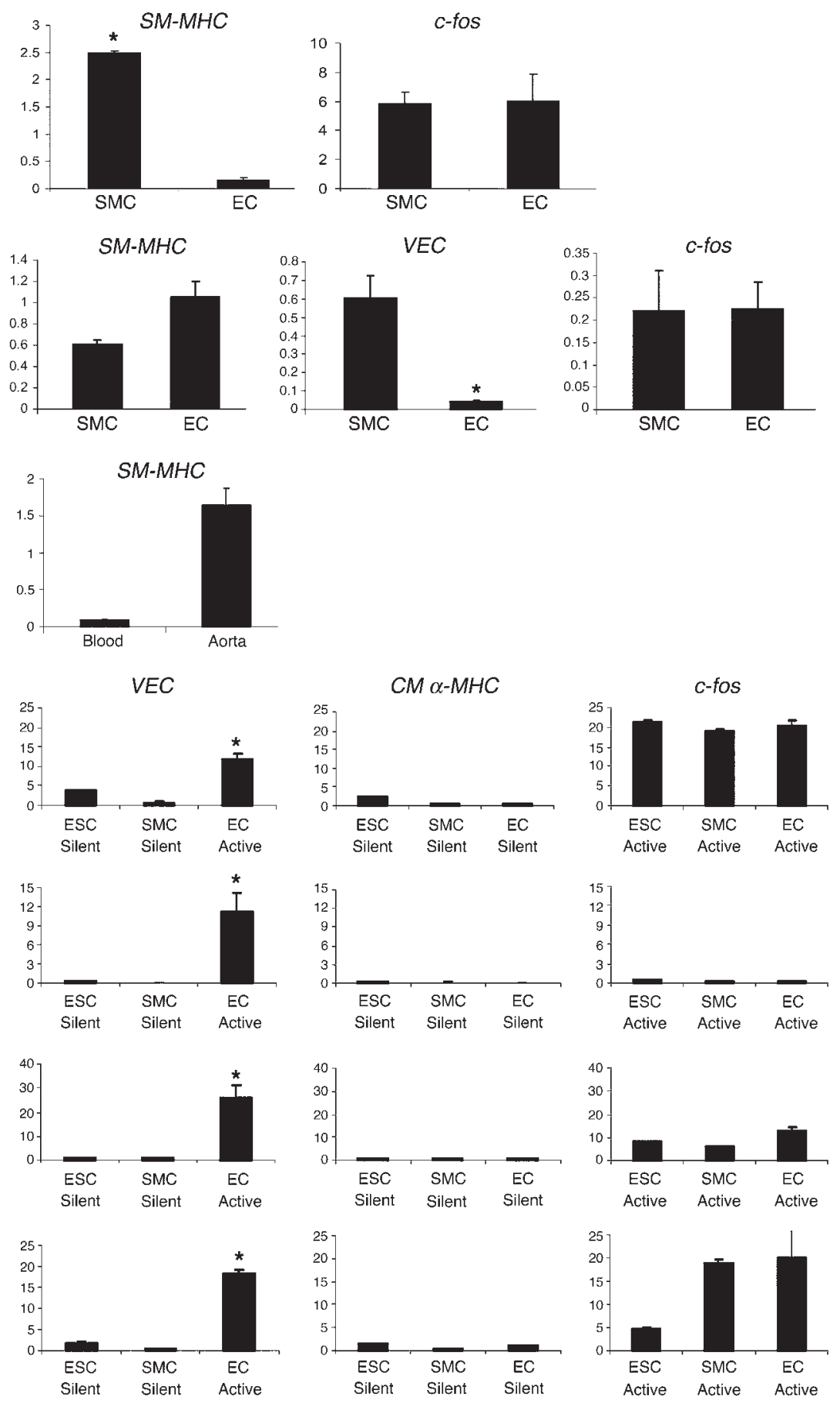

Figure 1

Cell-specific histone modifications correlate with cell-selective binding of SRF to $\alpha$-SMA and SM-MHC. (A) Quantitative ChIP analysis for SRF enrichment at the 5'-CArG boxes of $\alpha$-SMA, SM-MHC, and $c$-fos from chromatin isolated from rat aortic SMCs and ECs. (B) Chromatin isolated from SMCs and ECs was digested into mononucleosomal fragments by micrococcal (S7) nuclease. DNA was purified and amplified with primers flanking the 5'-CArG boxes of $\alpha$-SMA, SM-MHC, and c-fos. The 5'-promoter region of the EC-specific gene VEC was also included as a control. The VEC promoter does not contain CArG boxes. See Methods for explanation. (C) Chromatin was isolated from rat aortas and blood and SRF binding measured by ChIP as in A. (D) Histone modifications that have been associated with activation of gene expression (25) were measured at $\alpha$-SMA, VEC, cardiac-muscle myosin heavy chain (cardiac-specific; $C M \alpha-M H C$ ), and $c$-fos promoters by ChIP in cultured ES cells (ESC), SMCs, and ECs. We observed patterns similar to $\alpha-S M A$ at SM-MHC (data not shown). All ChIP data for Figure 1 and other figures are plotted as fold enrichment over equivalent amounts of input DNA, and Figure 1D is scaled equally on each horizontal panel (i.e., for each modification) so that enrichments between each of the different promoters can be compared. ${ }^{*} P<0.05$ measured by Student's $t$ test. Controls with beads only and without antibody consistently failed to immunoprecipitate DNA that amplified by real-time PCR (data not shown; see Supplemental Figure 2) for this and all other ChIP experiments. H3K79dMe, H3 Lys79 di-methylation; H3K9Ac, H3 Lys9 acetylation. 
matin. The nucleosome is composed of $146 \mathrm{bp}$ of genomic DNA wrapped around an octamer of histone proteins ( 2 copies each of histones H2A, H2B, H3, and H4). Importantly, chromatin structure and function can be profoundly influenced by posttranslational modifications to the histone proteins within the nucleosomes, such as acetylation and methylation of histone lysine residues by the action of nuclear proteins with histone acetyltransferase (HAT) or histone methyltransferase (HMT) activity (17). It is well recognized that histone modifications to nucleosomes play fundamental roles in determining how permissive promoter chromatin is for binding of transcription factors to DNA, thereby playing a critical regulatory role in transcriptional regulation.

Modifications to histones within chromatin have been shown to regulate how permissive chromatin is to transcription factor DNA binding by 2 general mechanisms $(16,17)$. First, histone acetylation has been shown to relieve structural chromatin compaction, possibly through disruption of interactions between adjacent nucleosomes or by loosening contacts between histones and DNA (18-21). This has the effect of making the DNA template itself more accessible to proteins that require contact with the DNA template, such as DNA-binding transcription factors. Second, methylated histones within chromatin can serve as docking sites for transcription factors, thereby directly tethering them to the DNA template (22). Although it is unclear whether these mechanisms operate in SMCs, we and others have recently documented that histone acetylation accompanies SRF binding to SMC promoters in SMCs $(11,12,23)$, suggesting that epigenetic modification of chromatin structure may indeed play a role in controlling SRF binding. We therefore further investigated the histone modification status of SMC gene promoters in this study; whether these modifications might be utilized to control SRF binding to chromatin by proteins that profoundly activate (myocardin) or repress (Kruppel-like factor 4 [KLF4]) SMC gene expression; and whether these processes contribute to the repair of vascular injury in vivo.

\section{Results}

SRF selectively associated with SMC gene CArG box chromatin in SMCs versus non-SMCs. We first tested whether SRF binding to CArG boxes of SM genes was selective for SMCs, utilizing quantitative chromatin immunoprecipitation (ChIP) assays. This assay analyzes SRF binding to endogenous DNA sequences within native chromatin by formaldehyde fixation of cells followed by immunoprecipitation of SRF and PCR analysis of DNA from SRFbound chromatin. In contrast to previous studies utilizing electrophoretic mobility shift assays (EMSAs), a method that analyzes binding of SRF to naked DNA probes that are not wrapped into chromatin (24), we found much higher levels of SRF binding to SMC CArG boxes in cultured SMCs as compared with non-SMCs, including ECs (Figure 1A and ref. 11). In addition, we found that these CArG box DNA sequences were more accessible within chromatin isolated from rat aortic SMCs than from ECs, as assayed by micrococcal (S7) nuclease digestion experiments (Figure 1B; see figure legend for interpretation of data). In contrast, SRF binding and chromatin accessibility were equivalent in SMCs and ECs at the 5 '-CArG box of $c$-fos, a gene expressed in both cell types. The selectivity of SRF for SMC gene chromatin in SMCs was confirmed in vivo, as SRF was also enriched at SMC gene promoters based on comparison of ChIP analysis of chromatin isolated from rat aorta and rat blood cells (Figure 1C). Because SRF selectively bound to chromatin isolated from SMCs and not non-SMCs, these findings suggested that epigenetic modifications of chromatin structure might regulate SMC-specific SRF binding to CArG boxes, possibly through regulation of CArG box accessibility to SRF.

SMC gene CArG boxes exhibited cell-specific histone modification patterns. Acetylation and methylation to histone $\mathrm{H} 3$ and $\mathrm{H} 4$ lysine residues within chromatin play an essential epigenetic role in eukaryotic transcriptional regulation $(16,17)$. To begin to determine the potential role of these modifications in control of SMC gene expression, we examined $\alpha-S M A$ and $S M-M H C$ promoter CArG box regions for the presence/absence of well-studied histone $\mathrm{H} 3 / \mathrm{H} 4$ lysine modifications (see Table 1) in multiple in vitro cell differentiation/lineage models including mouse ES cells, rat aortic SMCs, rat aortic ECs, rat L6 skeletal muscle myotubes, SMCs purified from mouse embryoid bodies, and mouse A404 SMCs, as well as in rat aorta and blood cells in vivo. We measured levels of H3 Lys4 di-methylation (H3K4dMe), H3 Lys9 acetylation, H3 Lys79 di-methylation, and $\mathrm{H} 4$ acetylation (H4Ac) by ChIP with antibodies specific to these modifications. The presence of each of these modifications has been correlated with transcriptional competence in other multicellular eukaryotic cell systems (25), although the mechanisms whereby they contribute to transcriptional activation are poorly understood. Of major interest, these modifications were highly enriched at the promoter CArG boxes of $\alpha$-SMA and SM-MHC in SMCs, but not in the non-SMCs we tested (Figure 1D and Supplemental Figure 1; supplemental material available online with this article; doi:10.1172/JCI26505DS1). Therefore, the histone modification patterns at these promoters distinguished SMCs from non-SMCs and vice-versa and correlated closely with the ability of SRF to selectively bind CArG box chromatin in SMCs (Table 1).

The $\alpha$-SMA promoter-enhancer exhibited locus-specific histone modification patterns. Results outlined in Table 1 demonstrate that the SMC genes examined in this study display SMC-specific histone modifications at the 5'-CArG boxes. To determine whether these modifications were specific to the chromosomal locus surrounding the CArG regions as opposed to reflecting a nonspecific general pattern over a chromosomal region that extended beyond the 5 '-CArG boxes, we mapped the histone modifications represented in Table 1 across approximately $6 \mathrm{~kb}$ of the $\alpha$-SMA promoterenhancer locus with PCR primers placed at 400-bp intervals by ChIP assays. Figure 2 demonstrates that enrichment of $\mathrm{H} 4$ and $\mathrm{H} 3$ acetylation (Table 1) were relatively low from positions $-2,800$ to $-1,600$ in the $5^{\prime}$ region. However, at position $-1,600$ to $-1,200$, there was a sharp rise in these modifications, which was increased even further at +400 in the coding region. We observed similar patterns for H3K4dMe and H3 Lys79 di-methylation (data not shown).

\section{Table 1}

Patterns of histone modification at CArG boxes of SMC gene promoters

$\begin{array}{lcc}\text { Histone modification } & \text { Non-SMCs } & \text { SMCs } \\ \text { H3K4dMe } & \text { Absent } & \text { Present } \\ \text { H3 Lys79 di-methylation } & \text { Absent } & \text { Present } \\ \text { H3 Lys9 acetylation } & \text { Absent } & \text { Present } \\ \text { H4Ac } & \text { Absent } & \text { Present } \\ \text { SRF binding } & \text { Absent } & \text { Present }\end{array}$

Non-SMCs include ES cells, ECs, blood cells, skeletal muscle myotubes, undifferentiated A404 SMC progenitors. 


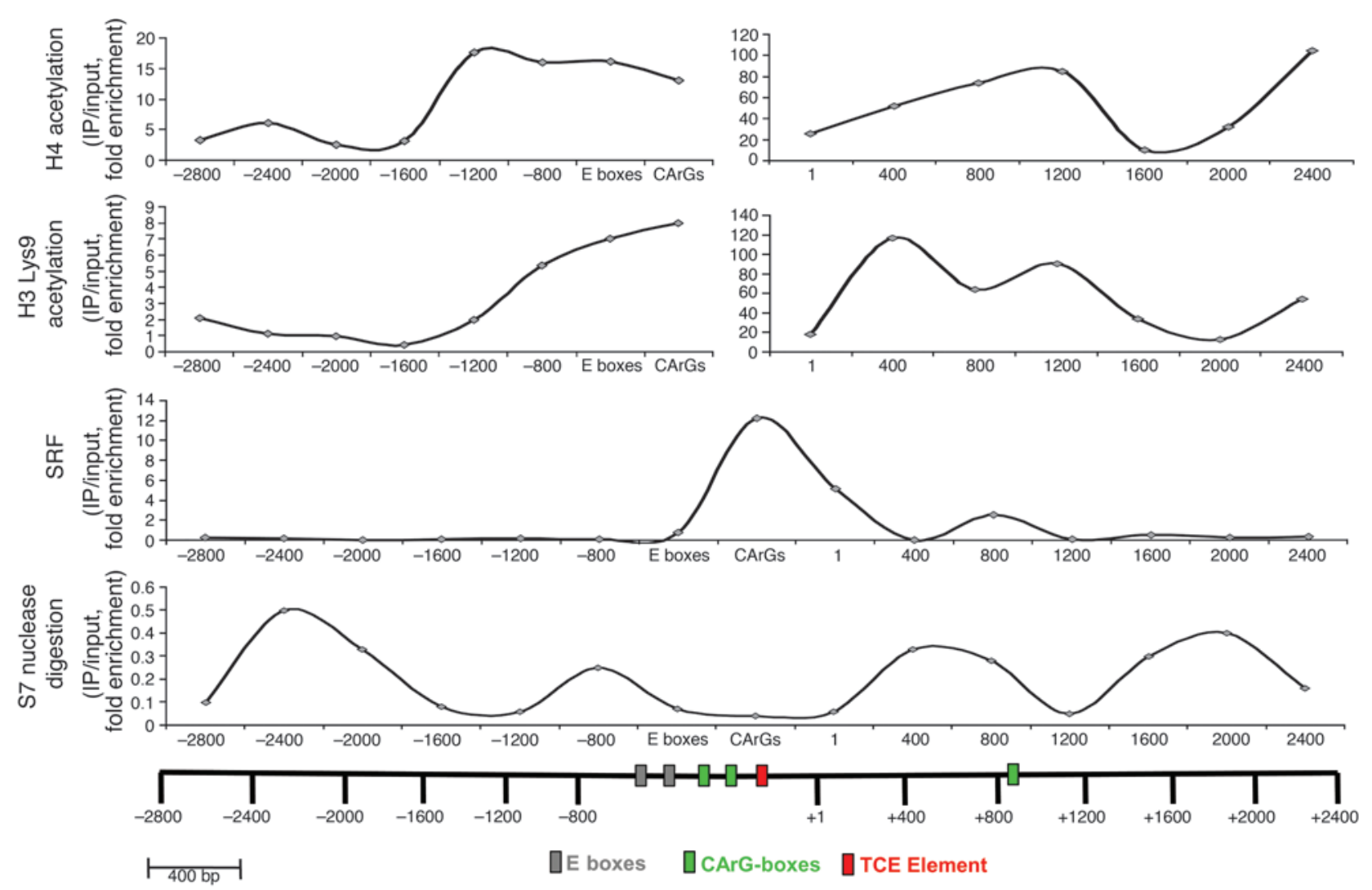

Figure 2

Characterization of transcription factor and histone modification distribution across the $\alpha$-SMA promoter-enhancer locus. Fifteen pairs of PCR primers spaced at 400-bp intervals were used in ChIP assays to map the distribution of factors plotted. Nuclease accessibility was determined as in Figure 1. E boxes are cis-elements $5^{\prime}$ to CArG boxes. Data are representative of 2 independent experiments.

SRF, TFIID, and RNA polymerase II displayed enrichments that were consistent with the positions of the CArG boxes, TATA box, and coding region, respectively (Figure 2 and data not shown). Finally, we observed micrococcal nuclease hypersensitivity at the 5 -CArG box region, consistent with the data from Figure 1 indicating that accessible chromatin is an important determinant for SRF binding to CArG box DNA. Thus, in addition to cell and gene specificity (see Table 1), these histone modifications also displayed locus specificity within the $\alpha$-SMA chromosomal region, further suggesting that these modifications may play an important role in SMC-selective transcription.

SMC-specific H3K4dMe and H4Ac were not dependent on SRF binding to $C A r G$ chromatin. The results described above provided us with correlative data linking histone modifications present within chromatin of SMC genes to SRF binding within this chromatin. We next sought to determine whether any of these modifications might be utilized by SRF to bind CArG box chromatin in SMCs. We began by examining which, if any, of the SMC-specific histone modifications (Table 1) were not dependent on SRF binding to CArG chromatin, as these could be important upstream regulators of SMC-selective SRF binding. To this end, we generated stable lines of cultured SMCs containing wild-type or CArG box mutant $\alpha$-SMA promoter-enhancer transgenes (see Methods and Figure 3A). We then assayed for SRF binding and histone modifications with ChIP PCR primers specific for either the transgene or the endogenous gene.
Although wild-type transgenes displayed enrichments of SRF and histone modification that were virtually identical to those of the endogenous locus, CArG mutants displayed complete loss of SRF binding with severely impaired H3 Lys9 acetylation and H3 Lys79 di-methylation (Figure 3A). These results indicated that the presence of these 2 modifications was dependent on intact CArG boxes that are capable of binding SRF and that they were thus likely enzymatically added to chromatin downstream of SRF binding during transcriptional activation. In contrast, levels of $\mathrm{H} 3 \mathrm{~K} 4 \mathrm{dMe}$ and $\mathrm{H} 4 \mathrm{Ac}$ at mutant CArG boxes remained at levels comparable to the endogenous locus, suggesting that the presence of these modifications was not dependent on intact CArG boxes or SRF-CArG occupancy. These result thus identified $\mathrm{H} 3 \mathrm{~K} 4 \mathrm{dMe}$ and $\mathrm{H} 4 \mathrm{Ac}$ as potential upstream regulators of SMCselective SRF binding to SMC genes. Because of this, we chose to focus on the function of these 2 histone modifications in relation to SRF binding to CArG box chromatin. Since we recently identified myocardin and KLF4 as factors that potentially regulate SRF binding to CArG box chromatin $(10,13)$, we investigated further whether these proteins influence the chromatin-binding properties of SRF and whether H3K4dMe and/or H4Ac might play a role in this process in SMCs.

Myocardin interacted with H3K4dMe and selectively enhanced SRF binding to CArG box chromatin of SMC genes. Myocardin is a musclerestricted SRF coactivator that forms a ternary complex with SRF to activate SMC gene expression (7). Although myocardin does not 
A
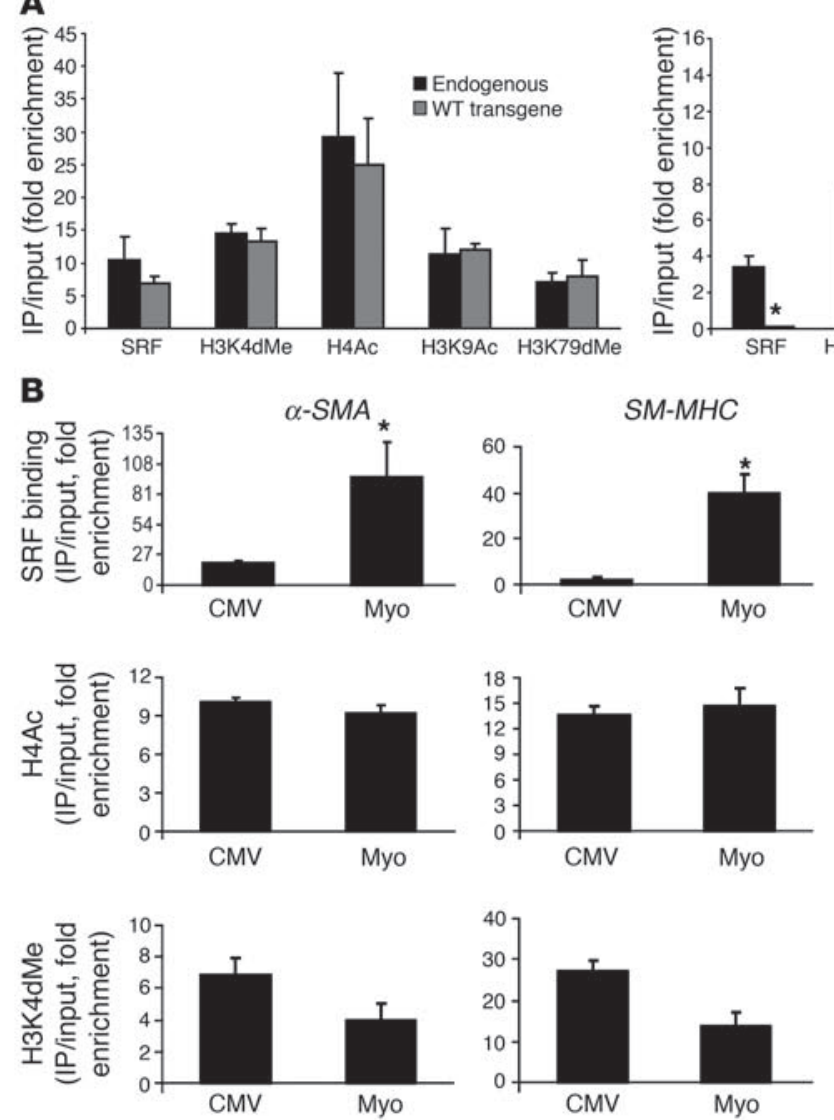

C
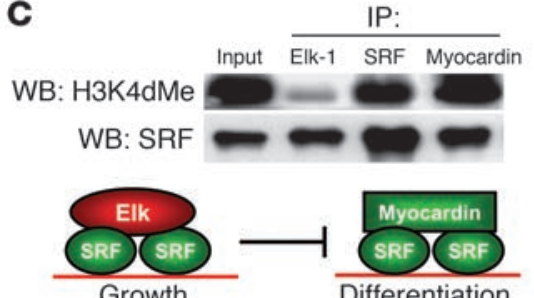
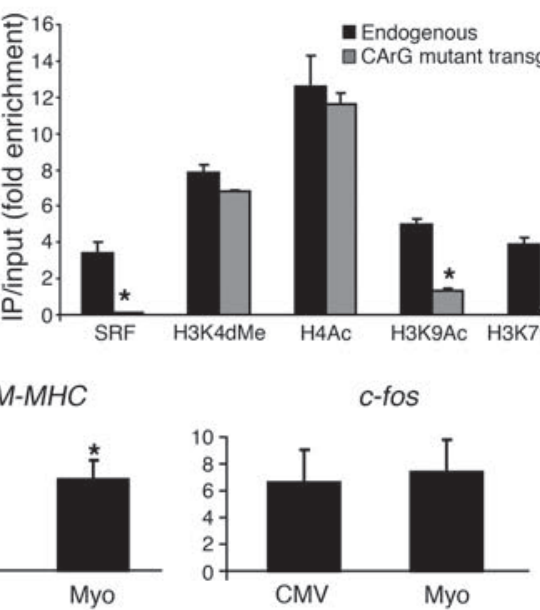

E

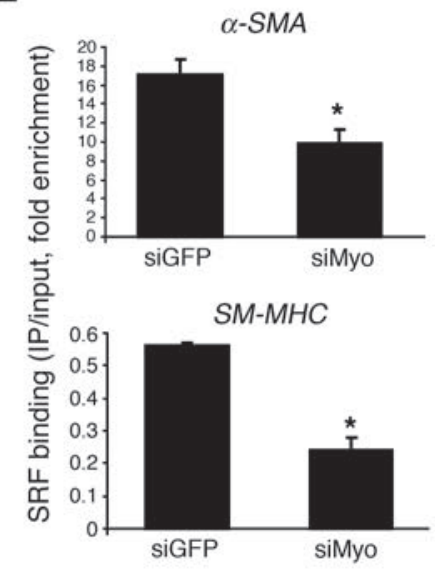

F
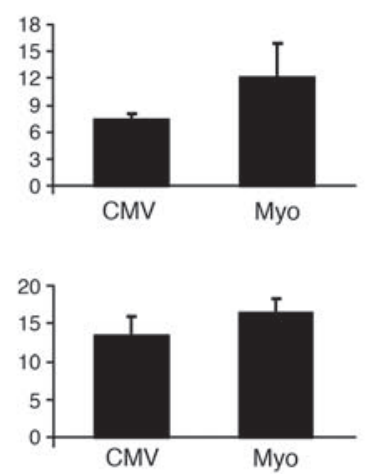

D SRF IP $\frac{\text { Adenovirus }}{\text { CMV Myo }}$

WB: H3K4dMe

WB: SRF

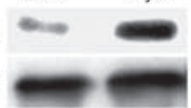

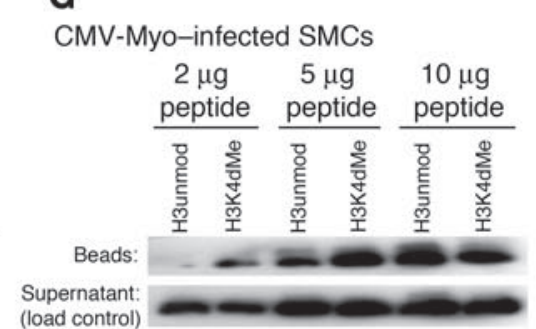

H CMV-Myo-infected SMCs

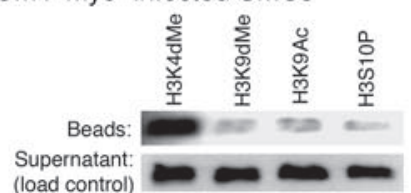

Figure 3

Identification modifications that contribute to myocardin/SRF binding to CArG boxes. (A) $\alpha$-SMA transgenes were stably transfected into rat aortic SMCs by the integrase system as described in Methods, and ChIP was performed with primers specific for the transgene and the endogenous gene. ${ }^{*} P<0.05$ by Student's $t$ test. (B) SMCs were infected with adenovirus harboring CMV-myocardin (Myo) or CMV-empty (CMV) expression vectors, and ChIP was performed for SRF, H4Ac, and H3K4dMe. (C) SMCs were infected with adenovirus as in B. Elk-1, SRF, and FLAG-myocardin immunoprecipitates were subjected to Western blotting for H3K4dMe and SRF. Nonimmune IgG antisera failed to immunoprecipitate SRF and H3K4dMe from SMC extracts in these and all other protein IP experiments (data not shown and Supplemental Figure 2). (D) SMCs were infected as in B, and SRF immunoprecipitates were subjected to Western blotting for H3K4dMe and SRF. (E) SMCs were infected with adenoviruses expressing siRNAs to myocardin (siMyo) or GFP (siGFP; control). Chromatin was isolated, and ChIP measured levels of SRF binding to 5'-CArG boxes. (F) SMCs were infected as in E, and nuclear extracts were treated as in D. (G) Peptide binding assay with FLAG-myocardin as described in Methods. FLAG-myocardin immunoprecipitates collected from SMC extracts containing the corresponding biotinylated peptides were subjected to Western blotting using HRP-streptavidin. H3unmod, unmodified H3 peptide. (H) Myocardin peptide binding assay as in G, comparing the ability of myocardin to immunoprecipitate $2 \mu \mathrm{g}$ of H3 peptides di-methylated at Lys4 (H3K4dMe), di-methylated at Lys9 (H3K9dMe), acetylated at Lys9, or phosphorylated at serine 10 (H3S1OP).

bind DNA or influence the DNA binding properties of SRF based on EMSA (26), we previously showed that myocardin selectively increased SRF binding to the CArG box region of $\alpha$-SMA but not c-fos within intact chromatin (10). We therefore investigated whether myocardin might utilize $\mathrm{H} 3 \mathrm{~K} 4 \mathrm{dMe}$ and/or H4Ac to enhance SRF binding to CArG box chromatin.
We began by infecting cultured rat aortic SMCs with an adenovirus expressing myocardin and then measured levels of SRF binding, H4Ac, and H3K4dMe at the $5^{\prime}$-CArG boxes of $\alpha$-SMA, $S M-M H C$, and $c$-fos by ChIP (Figure 3B). Myocardin greatly increased SRF association with CArG boxes at $\alpha$-SMA and $S M-M H C$, but not $c$-fos. H3 Lys9 acetylation was also increased at 
$\alpha-S M A$ and $S M-M H C$ (data not shown), consistent with recruitment of the H3 HAT p300 by myocardin (23). However, myocardin did not increase levels of $\mathrm{H} 4 \mathrm{Ac}$ or $\mathrm{H} 3 \mathrm{~K} 4 \mathrm{dMe}$ at $\alpha-S M A$ and SM-MHC. These results suggested that myocardin-induced enrichment of SRF binding to CArG boxes within intact chromatin involves some mechanism other than recruitment of histone H4 HAT or H3 Lys4 HMT activity to this promoter region.

An alternative is that myocardin/SRF complexes might interact with $\mathrm{H} 3 \mathrm{~K} 4 \mathrm{dMe}$, similar to other multiprotein complexes that dock to $\mathrm{H} 3 \mathrm{~K} 4 \mathrm{dMe}$ present within promoter chromatin during transcriptional activation in other cell systems $(22,27,28)$. To address this possibility, we first tested whether myocardin and/ or SRF could immunoprecipitate H3K4dMe from SMC nuclear extracts. Figure $3 \mathrm{C}$ demonstrates that both SRF and virally delivered FLAG-tagged myocardin were able to immunoprecipitate H3K4dMe from SMCs. Elk-1, which also forms a ternary complex with SRF distinct from that of myocardin (9), did not immunoprecipitate significant amounts of $\mathrm{H} 3 \mathrm{~K} 4 \mathrm{dMe}$, although it did immunoprecipitate SRF, indicating that the ability of SRF to immunoprecipitate H3K4dMe was specific to myocardin/SRF complexes. In addition, SRF was able to immunoprecipitate more $\mathrm{H} 3 \mathrm{~K} 4 \mathrm{dMe}$ from SMCs infected with myocardin-expressing adenoviruses than from control cells (Figure 3D), and adenovirally delivered siRNAs to myocardin reduced SRF binding to $\alpha-S M A$ and $S M-M H C$ CArG box chromatin (Figure 3E) and interfered with the ability of SRF to immunoprecipitate $\mathrm{H} 3 \mathrm{~K} 4 \mathrm{dMe}$ from SMCs (Figure $3 \mathrm{~F}$ ). We conclude that myocardin/SRF complexes physically interact with $\mathrm{H} 3 \mathrm{~K} 4 \mathrm{dMe}$ and that the interaction of SRF with CArG box chromatin and $\mathrm{H} 3 \mathrm{~K} 4 \mathrm{dMe}$ is sensitive to expression levels of myocardin.

To directly determine whether the interaction of myocardin with $\mathrm{H} 3 \mathrm{~K} 4 \mathrm{dMe}$ was specific, we incubated SMC nuclear extracts expressing FLAG-tagged myocardin with increasing concentrations of biotinylated peptides composed of residues 1-21 of histone $\mathrm{H} 3$ that were either di-methylated at Lys 4 or unmodified. Myocardin was then immunoprecipitated from these extracts, and immunoprecipitates were subjected to Western blotting with HRP-conjugated streptavidin. These are standard assays for examination of whether a transcription factor can physically associate with modified histones $(22,28)$. Figure $3 \mathrm{G}$ shows that myocardin preferentially bound $\mathrm{H} 3$ peptides di-methylated at Lys 4 rather than unmethylated $\mathrm{H} 3$ peptides at peptide concentrations ( 2 and $5 \mu \mathrm{g})$ below saturation $(10 \mu \mathrm{g})$. The preference of myocardin for $\mathrm{H} 3 \mathrm{~K} 4 \mathrm{dMe}$ was specific to this modification, as myocardin bound $\mathrm{H} 3 \mathrm{~K} 4 \mathrm{dMe}$ peptides much more effectively than biotinylated $\mathrm{H} 3$ peptides methylated at Lys9, acetylated at Lys9, or phosphorylated at Ser10, as depicted in Figure $3 \mathrm{H}$.

KLF4 antagonized myocardin through disruption of H4Ac and loss of $S R F$ binding to $C A r G$ box chromatin of SMC genes. Kruppel-like transcription factors play important roles in cardiovascular pathology (29). We recently showed, by transient transfection assays in cultured SMCs, that KLF4 could potently inhibit myocardininduced activation of SMC genes (13). The mechanisms of this effect are unknown. However, KLF4 specifically reduced SRF binding to $\alpha$-SMA CArG box chromatin by ChIP, but not EMSA (13), suggesting that an epigenetic component of chromatin might play a role in the KLF4 response. We therefore investigated whether KLF4 altered H4Ac and/or H3K4dMe levels at CArG box chromatin by ChIP (Figure 4).

Virally delivered KLF4 induced large reductions in SRF binding at the CArG boxes of $\alpha$-SMA and SM-MHC in SMCs (Figure 4A).
Although KLF4 had no effect on levels of H3K4dMe at these promoters and was unable to immunoprecipitate $\mathrm{H} 3 \mathrm{~K} 4 \mathrm{dMe}$ (data not shown), SMCs expressing KLF4 displayed marked reductions in $\mathrm{H} 4 \mathrm{Ac}$ at $\alpha$-SMA and MHC. Consistent with the notion that H4Ac is an important determinant of chromatin accessibility, KLF4 decreased micrococcal nuclease sensitivity (Figure 4A) at the CArG boxes of $\alpha$-SMA and SM-MHC but not c-fos. Loss of H4Ac was also accompanied by the appearance of the $\mathrm{H} 4$ deacetylase HDAC2, and similar to other studies (30), we were able to detect an interaction between KLF4 and HDAC2 within SMC extracts (Supplemental Figure 3). Strikingly, even the potent ability of myocardin adenovirus to enhance SRF-CArG association within chromatin was inhibited by coinfection of KLF4 adenoviruses (Figure 4B), suggesting that KLF4 might counteract the pro-myogenic effects of myocardin on SMC gene expression by blocking the ability of myocardin to enhance SRF binding to CArG box chromatin, possibly due to deacetylation of histone $\mathrm{H} 4$.

KLF4 can bind to evolutionarily conserved TGF- $\beta$ (TCE) DNA sequences adjacent to CArG boxes of SM gene promoters $(31,32)$, suggesting that KLF4 might directly recruit histone H4 deacetylase activity to these promoters to induce chromatin compaction, thereby rendering CArG box chromatin inaccessible to SRF. To determine whether KLF4-induced loss of SRF binding from SMC gene CArG regions was dependent on histone deacetylase (HDAC) activity, we treated SMCs with trichostatin A (TSA), a standard reagent used to inhibit HDAC activity. Treatment of SMCs with TSA completely blocked the ability of KLF4 to induce loss of $\mathrm{H} 4 \mathrm{Ac}$ at $\alpha$-SMA and partially inhibited this effect at $S M-M H C$, in a dose-dependent manner (Figure 4C). TSA also restored nuclease accessibility to these promoters (data not shown). However, mRNA levels and SRF binding were not restored despite the reappearance of H4Ac. Because KLF4 suppresses expression of myocardin (13), we postulated that absence of myocardin might be responsible for the failure of SRF to bind in the presence of KLF4 plus TSA. Figure 4D demonstrates that myocardin expression remained repressed in response to KLF4 in the presence of TSA, indicating that KLF4 inhibits expression of endogenous myocardin by an HDAC-independent mechanism. Consistent with suppression of myocardin, KLF4 also inhibited the ability of SRF to immunoprecipitate H3K4dMe (Figure 4E). Since data presented in Figure 3 indicates that myocardin influences the ability of SRF to efficiently associate with $\mathrm{H} 3 \mathrm{~K} 4 \mathrm{dMe}$ and CArG box chromatin, we tested whether introducing myocardin back into TSA-treated SMCs expressing KLF4 would reestablish SRF binding to CArG box chromatin, due to the restoration of H4Ac combined with reintroduction of myocardin. Indeed, TSA treatment of SMCs coinfected with myocardin and KLF4 adenoviruses restored the ability of myocardin to enhance mRNA expression and SRF binding to CArG chromatin of $\alpha$-SMA and partially restored binding of SRF at SM-MHC (Figure 4F).

Myocardin and KLF4 exerted opposing influences over SRF-CArG binding to SMC genes in vivo. To test whether myocardin could activate SMC gene expression through increases in SRF binding in vivo, we injected tail veins of transgenic mice carrying $S M-M H C$ promoter-enhancers driving SMC-specific expression of LacZ with myocardin adenoviruses or control viruses (Figure 5). Injection of virus into mouse tail veins resulted in uptake of virus by the liver via entry into the portal circulation, and mRNA levels of myocardin measured from livers of injected mice confirmed that adenoviral delivery was successful (Figure 5A). Figure 5B shows 
A
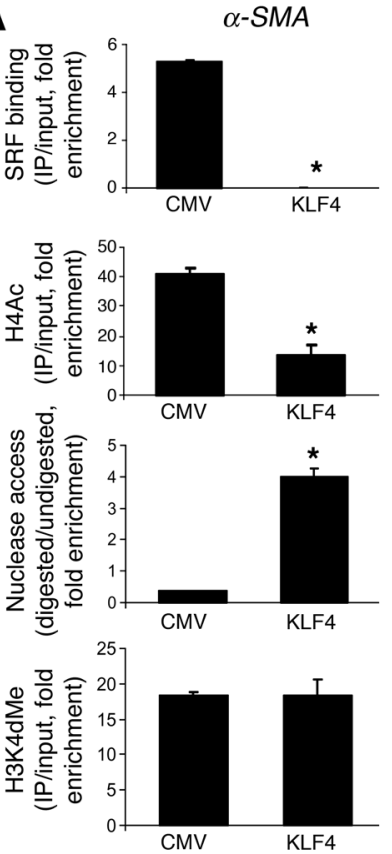

B

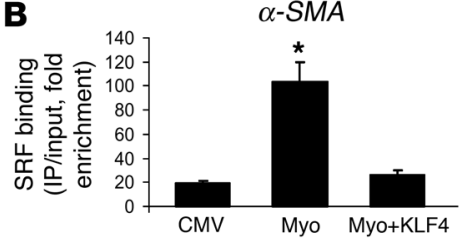

C
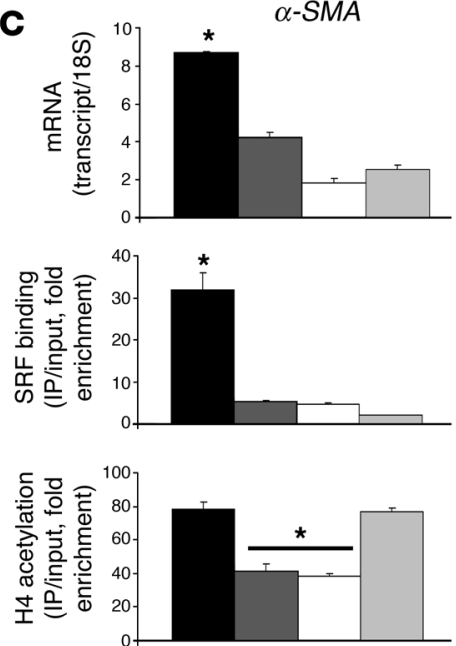
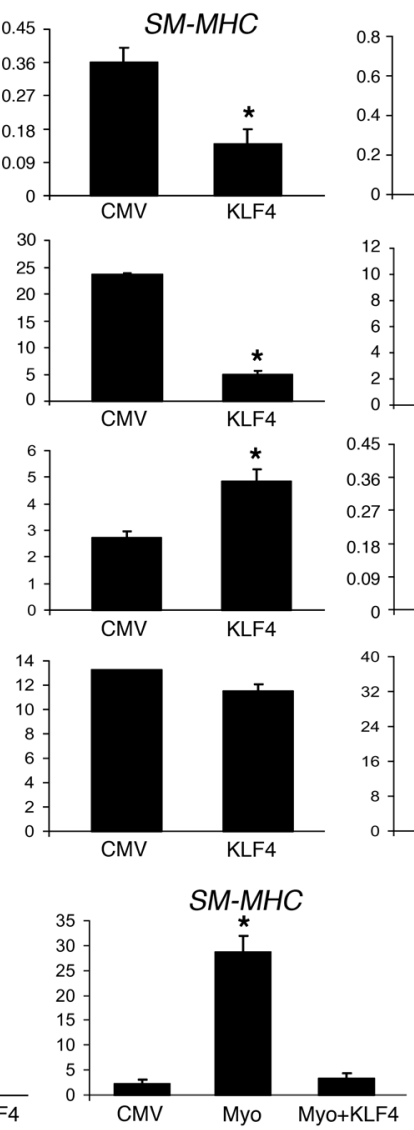

$\mathbf{F}$

F
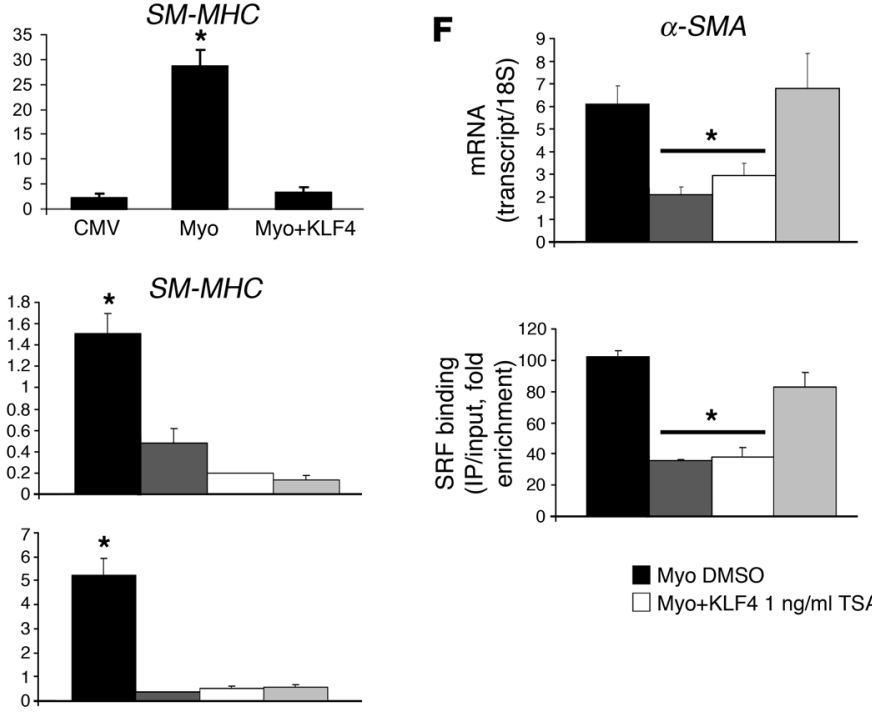

Myo DMSO SRF IP $\frac{\text { Adenovirus }}{\text { CMV KLF4 }}$
WB: H3K4dMe
WB: SRF
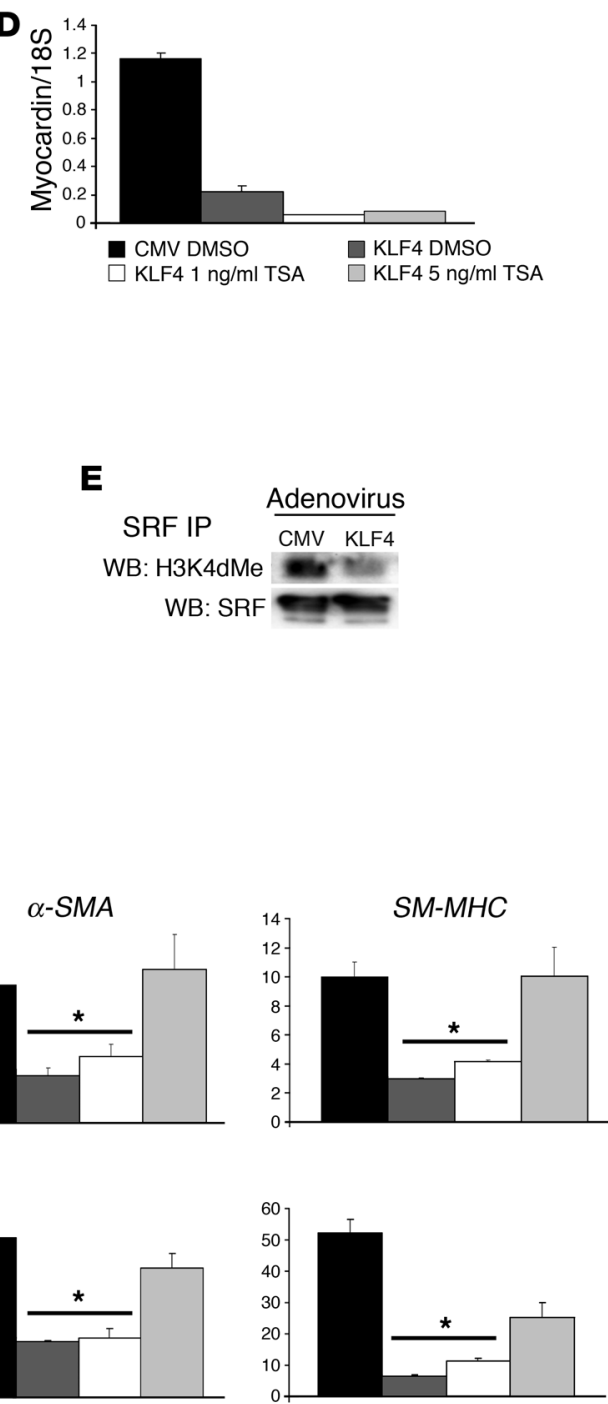

$\square$ Myo+KLF4 DMSO $\square$ Myo+KLF4 5 ng/ml TSA

Figure 4

KLF4 promotes loss of SRF binding and H4Ac at $\alpha$-SMA and SM-MHC. (A) SMCs were infected with CMV-KLF4 (KLF4) or CMV-empty adenoviruses and SRF, H4Ac, and H3K4dMe measured by ChIP. ${ }^{*} P<0.05$ by Student's $t$ test. Accessibility to micrococcal nuclease digestion was measured as in Figure 1B. (B) Cultured SMCs were infected with equivalent amounts of CMV-empty, CMV-myocardin, or CMV-myocardin with CMVKLF4 (myo+KLF4) adenoviruses and SRF binding to CArG boxes measured by ChIP as above. (C, D, and F) ChIP and real-time RT-PCR were measured from cultured SMCs infected with the corresponding adenoviruses with TSA dissolved in DMSO at $1 \mathrm{ng} / \mathrm{ml}$ or $5 \mathrm{ng} / \mathrm{ml}$ or in DMSO only. ${ }^{*} P<0.05$ for samples when compared with CMV-empty control cells by Student's $t$ test. (E) SRF was immunoprecipitated from SMCs infected with CMV-KLF4 or control (CMV) viruses, and immunoprecipitates were subjected to Western blotting for H3K4dMe and SRF (IP and IP control).

that myocardin was able to robustly activate transcription of otherwise silent SM-MHC promoter transgenes in mouse liver, as evidenced by strong staining of $\mathrm{LacZ}$ in livers from mice injected with myocardin virus. In addition, we observed dramatic increases in expression of endogenous $\alpha-S M A$ and SM-MHC mRNA, accompanied by increases in SRF binding to the CArG boxes of 
A
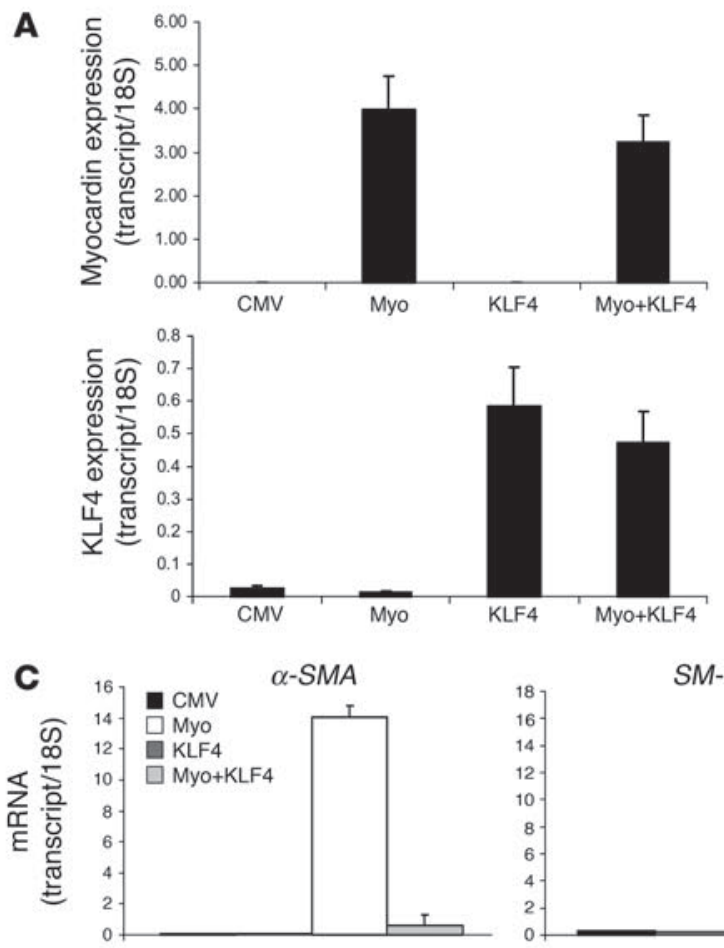

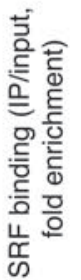
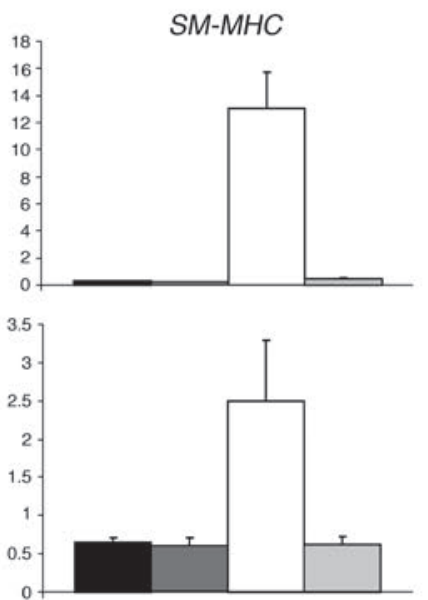

B

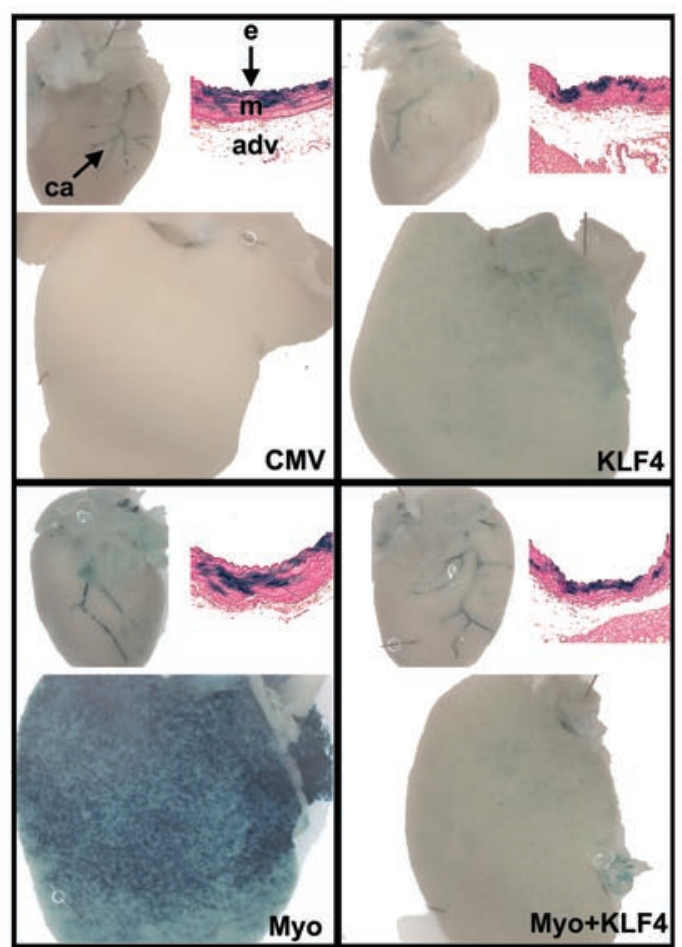

Figure 5

Myocardin and KLF4 exert opposing influences over SMC gene expression in transgenic mouse liver in vivo. (A) mRNA was extracted from liver in mice infected with CMV-empty control viruses, CMV-KLF4, CMV-myocardin, or mice coinjected with myocardin and KLF4 viruses. Expression levels of myocardin and KLF4 were measured by real-time PCR to document that delivery of these genes was successful. Data were normalized to levels of 18 S expression. (B) LacZ staining from SM-MHC-LacZ-transgenic mice injected as in A. For each panel in B, the top left image shows hearts expressing SMC-specific LacZ staining in coronary arteries (ca), and the top right image shows cross-sections taken from these mice displaying SMC-specific LacZ staining in the media $(\mathrm{m})$ of aortas. The endothelial layer (e) and adventitia (adv) are labeled. The media exhibit mosaic staining, which is typical for $S M-M H C$-transgenic mice. The bottom image in each panel shows staining for the presence of LacZ in mouse liver. (C) mRNA levels of $\alpha-S M A$ and SM-MHC were measured by real-time RT-PCR, and SRF binding to CArG box chromatin of these genes was measured by ChIP, in livers of mice injected with the indicated corresponding adenoviruses.

these genes by ChIP in response to myocardin (Figure 5C). We also observed enhanced SRF binding in cultured rat aortic ECs expressing myocardin (Figure 6B), suggesting that the positive influence of virally delivered myocardin on SRF binding to CArG box chromatin is operational during activation of SMC genes in non-SMCs in culture as well as in vivo.

We next tested whether KLF4 could inhibit the ability of myocardin to induce SRF binding and increase expression of $\alpha$-SMA and $S M-M H C$ in vivo. Remarkably, coinjection of KLF4 with myocardin adenoviruses abolished myocardin-induced LacZ staining, mRNA expression, and SRF binding to CArG boxes of $\alpha$-SMA and SM-MHC in mouse liver (Figure 5, B and C). KLF4 was also able to inhibit myocardin-induced SRF binding to CArG boxes in cultured ECs (Figure 6C), indicating that KLF4 could antagonize myocardin activity in cultured SMCs (Figure 4B) and cultured non-SMCs in vitro, similar to its effects in transgenic mouse liver in vivo. Taken together, these results in cultured ECs and transgenic mouse liver are surprising and could be the result of disrupted transcriptional circuitry in response to overexpression of these factors. Regardless of the mechanisms, we conclude that myocardin and KLF4 have the ability to exert opposing influences over SMC gene expression in vivo, at least in part through regulation of SRF association with CArG box chromatin of SMC genes.

SRF binding to CArG box chromatin was disrupted at SMC genes in response to $P D G F-B B$ in culture and after vascular injury in vivo. PDGF$\mathrm{BB}$ is a well-characterized signaling molecule that induces phenotypic switching of cultured SMCs, including profound transcriptional repression of SMC gene expression (1). We therefore investigated whether PDGF-BB might regulate the chromatin binding properties of SRF. As depicted in Figure 7A, 24 hours of PDGF-BB treatment increased expression of KLF4 and decreased expression of myocardin, relative to vehicle-treated control cells. 
This effect persisted to 72 hours of PDGF-BB treatment and was reversible upon replacement of PDGF-BB-containing media with vehicle at 24 hours and allowing cells to incubate for an additional 48 hours in vehicle media. The ability of SRF to immunoprecipitate H3K4dMe from PDGF-BB-treated cells was also diminished compared with vehicle-treated cells (Figure 7B), perhaps due to reductions in myocardin expression. We next investigated levels of SRF binding, H4Ac, and H3K4dMe by ChIP, as shown in Figure $7 C$. We observed reversible reductions in mRNA levels of $\alpha-S M A$ and $S M-M H C$ in response to PDGF-BB. Consistent with downregulation of myocardin and upregulation of KLF4 (see data in Figures 3 and 4), PDGF-BB induced decreases in SRF binding and $\mathrm{H} 4 \mathrm{Ac}$ at CArG boxes of $\alpha-S M A$ and $S M-M H C$. All of these effects were reversible upon replacement of PDGF-BB with vehicle.

To determine whether SRF binding to CArG chromatin plays a role during SMC phenotypic switching in vivo, we subjected rats to balloon catheter injury of carotid arteries (for mRNA) and aortas (for ChIP) and measured $\alpha-S M A$ and SM-MHC mRNA by RT-PCR and SRF binding and H4Ac by ChIP (Figure 7D). Vascular injury is characterized by reduced SMC gene expression (1), and we previously showed that KLF4 expression is upregulated (13), whereas myocardin expression is downregulated (10), under these conditions. mRNA levels of $\alpha-S M A$ and $S M-M H C$ were reduced at 24 hours after injury compared with sham control animals but returned to control levels 72 hours after injury, suggesting that rat vascular injury induces acute repression of SMC gene expression that is reversible at 3 days after injury, similar to the kinetics of medial SMC proliferation in this model (33). Importantly, levels of SRF binding and H4Ac changed in parallel with the changes in mRNA levels, indicating that control of SRF binding and H4Ac within CArG box chromatin may play a critical role during repression of SMC gene expression in response to vascular injury in vivo.

\section{Discussion}

The focus of the present study was to identify mechanisms that selectively regulate SRF binding to CArG box chromatin of SMC genes. Previous studies utilizing EMSA assays, which measured SRF binding to naked DNA templates not assembled into chromatin, demonstrated that SRF has the ability to bind to SMC gene promoter CArG box DNA sequences in virtually any cell type, including nonSMCs (11). However, our results using ChIP assays demonstrated that SRF binding to these sequences was restricted to SMCs within the context of native chromatin (Figure 1), suggesting that an epigenetic component of chromatin (e.g., histone modifica-

\section{Figure 6}

Myocardin and KLF4 exert opposing influences over SRF binding to SMC genes in cultured ECs. (A) Morphology of ECs infected with CMV-myocardin or control (CMV-empty) adenoviruses. (B) ChIP results for SRF binding to 5'-CArG boxes of the indicated genes from ECs infected with CMV-myocardin adenoviruses or control viruses. (C) ChIP as in B, in ECs infected with CMV-myocardin alone, controls, or ECs coinfected with CMV-myocardin and CMV-KLF4 viruses. ${ }^{*} P<0.05$ by student's $t$ test. tions) might be responsible for this activity. Table 1 outlines our results documenting the presence of SMC-specific histone modifications at SMC gene promoters that were utilized by myocardin and KLF4 to influence the chromatin-binding properties of SRF and hence regulate SMC gene expression. Collectively, the results of this study suggest a model whereby cell-specific epigenetic control mechanisms may influence cell-selective gene expression patterns important for regulation of cellular differentiation.

Although previous studies conclusively showed that myocardin did not influence the DNA binding properties of SRF to CArG box DNA templates by EMSA (26), results of the present studies clearly demonstrate that myocardin possesses the surprising ability to control SRF binding to CArG box DNA within intact chromatin by ChIP in cultured SMCs and non-SMCs in vitro and in transgenic mice in vivo. It is well established that SRF binds to CArG box DNA to recruit downstream accessory factors to regulate SMC transcription. However, our results add an unexpected layer of complexity to this model, in that an SRF accessory factor (e.g., myocardin) may also utilize a specific epigenetic element (H3K4dMe) to control SRF association with CArG box chromatin. That is, myocardin may utilize H3K4dMe present at SMC promoters to dock to chromatin, thereby tethering and/or stabilizing SRF binding to CArG box DNA sequences. Because H3K4dMe is present at SMC promoters in SMCs but not in non-SMCs, the presence of this modification in combination with muscle-restricted expression of myocardin may represent a mechanism whereby SRF can, in part, selectively bind CArG boxes of SMC genes in SMCs and not in non-SMCs to activate the SMC differentiation program. Direct confirmation of whether myocardin/SRF complexes utilize $\mathrm{H} 3 \mathrm{~K} 4 \mathrm{dMe}$ within CArG box chromatin will require identification of the HMT complex(es) that methylates SMC gene CArG chromatin and the development of in vitro chromatin reconstitution assays with SMC gene promoter-enhancer DNA wrapped into methylated nucleosomes that can accurately recapitulate in vivo
A

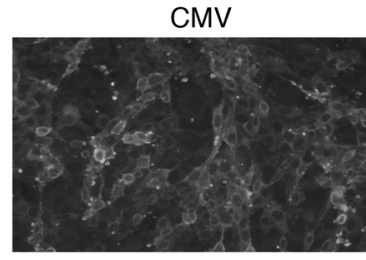

B

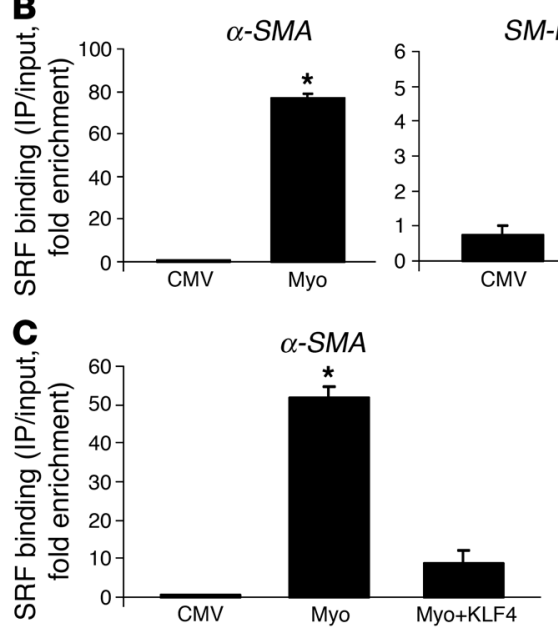

Myo

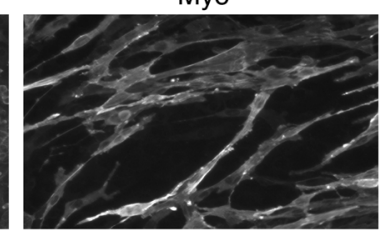

SM-MHC
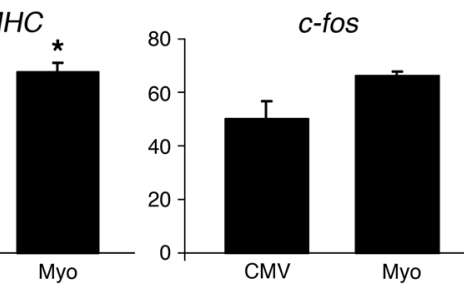

SM-MHC

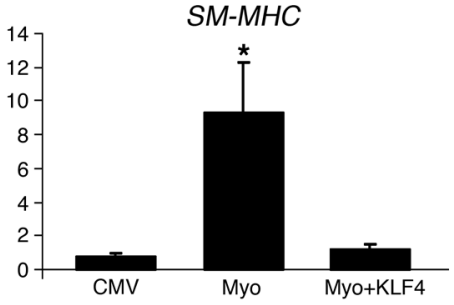


A
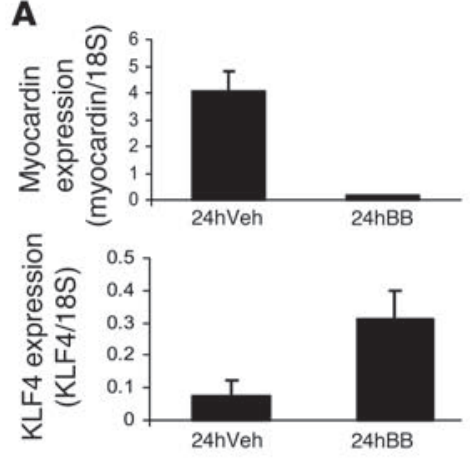

C
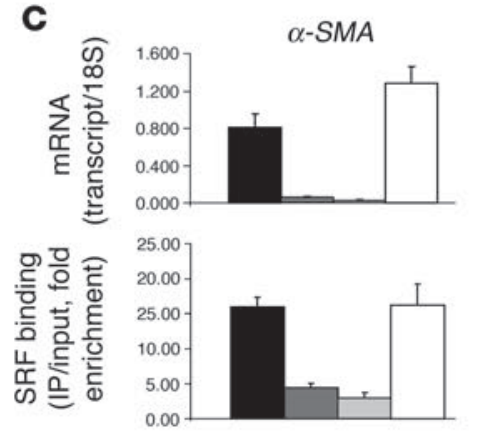

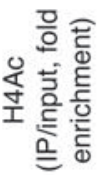

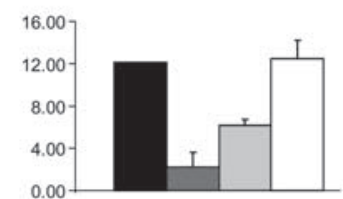

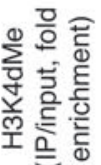

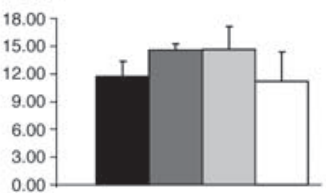

D
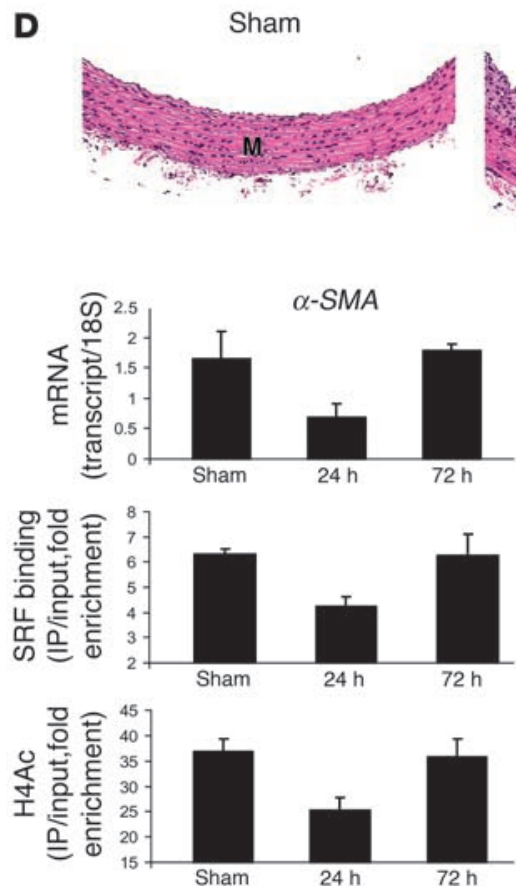

B

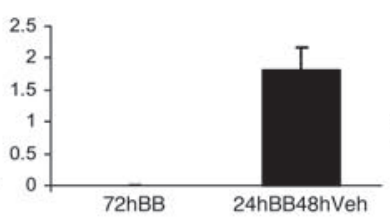

SRF IP
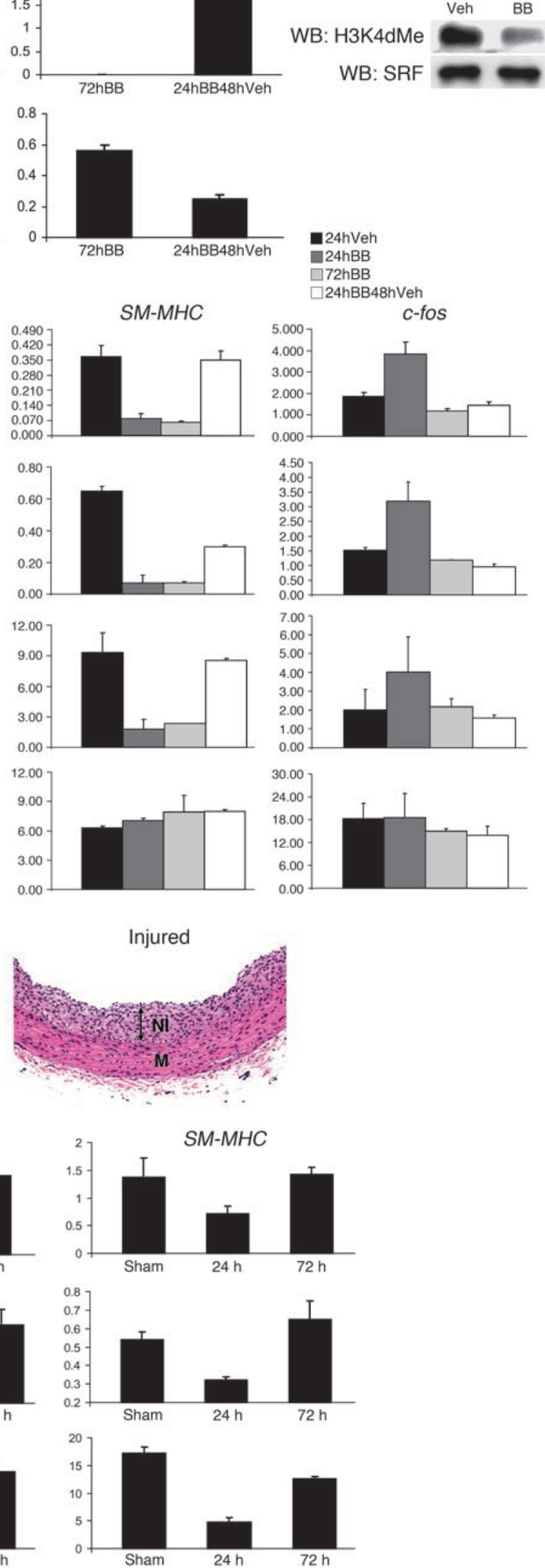

\section{Figure 7}

SRF binding to CArG box chromatin is disrupted during SMC phenotypic switching. (A) Left: Expression of myocardin and KLF4 in response to PDGF-BB (BB) treatment of cultured SMCs for 24 hours (24hBB) or vehicle treatment for 24 hours (24hVeh). Right: Similar data from cells treated with PDGF-BB for 72 hours (72hBB) or cells treated with PDGF-BB media for 24 hours, followed by replacement of PDGF-BB media with vehicle media and incubation for 48 hours (24hBB48hVeh). Data were normalized to expression of 18S. PDGF-BB treatments were performed as described previously (8). (B) SMCs were treated with vehicle (Veh) or PDGF-BB for 24 hours, and SRF immunoprecipitates were subjected to Western blotting for H3K4dMe or SRF (IP control). (C) SMCs were treated with PDGF-BB as in $\mathbf{A}$, mRNA was measured by RTPCR as in $\mathbf{A}$ (top panel), and ChIP was performed at the $5^{\prime}$-CArG regions with the indicated genes for the indicated parameters (SRF binding, etc). (D) Rats were injured with balloon catheter as described in Methods, and mRNA or chromatin was isolated and analyzed by real-time RT-PCR and ChIP, respectively. The top panel is a histological display of uninjured control aorta (Sham) and an injured aorta (Injured), demonstrating that the balloon catheter injury technique employed successfully injured the vessels, as indicated by the presence of a neointima (NI) 14 days after injury. $M$ refers to the position of the vessel media. For mRNA and ChIP (bottom panels), vessels were harvested 24 hours or 72 hours after injury and compared with control vessels. 
SMC transcription. Interestingly, preliminary data from other studies currently underway suggest that myocardin expression in cultured ECs and mouse liver can also establish the presence of $\mathrm{H} 3 \mathrm{~K} 4 \mathrm{dMe}$ and H4Ac at SMC gene promoters (O.G. McDonald and G.K. Owens, unpublished observations). Thus, we are currently investigating mechanisms whereby myocardin expression in cultured ECs and mouse liver might establish the presence of $\mathrm{H} 3 \mathrm{~K} 4 \mathrm{dMe}$ and H4Ac at SMC gene chromatin in other studies and whether the acquisition of $\mathrm{H} 3 \mathrm{~K} 4 \mathrm{dMe}$ and H4Ac in these nonSMCs might assist SRF binding to this chromatin.

We recently identified KLF4 as a potent suppressor of SMC differentiation. Those studies identified 2 mechanisms whereby KLF4 may repress SMC gene expression: (a) KLF4 virtually extinguished expression of endogenous myocardin and (b) KLF4 could directly inhibit activation of SMC genes by myocardin when these 2 proteins were coexpressed in transient transfection assays (13). The present studies demonstrate that the ability of KLF4 to directly inhibit myocardin-dependent activation of SMC gene expression is due, at least in part, to virtually complete abolishment of myocardin-induced increases in SRF binding to CArG box chromatin in cultured SMCs and non-SMCs in vitro and in transgenic mice in vivo. We also found this activity to be HDAC dependent, at least in cultured SMCs. It should be noted that our studies relied on KLF4 overexpression, and we could not compare protein levels of this factor under our experimental conditions with (patho)physiological protein levels due to lack of a suitable antibody, although mRNA expression levels are comparable (13). Thus, validation of this model will require the development of in vivo KLF4 loss-of-function experiments in SMCs, such as the use of mice harboring SMC-specific KLF4 conditional knockout or delivery of KLF4 siRNAs to the vasculature.

KLF4 may operate similarly to the recently described homeodomain-only protein HOP, which can directly oppose myocardin by recruiting HDAC2 to the $S M 22 \alpha$ promoter to promote deacetylation of histone $\mathrm{H} 4$ and transcriptional repression (34). Indeed, KLF4 can bind evolutionarily conserved TCE elements adjacent to CArG boxes in SMC promoters $(31,32)$ and can also interact with and recruit HDAC2 (ref. 30 and Supplemental Figure 3B), a class I HDAC that deacetylates histone H4 (35). Thus, our data support a model whereby KLF4 directly recruits HDAC activity to SMC genes to facilitate deacetylation of histone $\mathrm{H} 4$, chromatin compaction, and loss of myocardin/SRF binding in a TSA-sensitive manner. The increased nucleosomal/compacted character of the chromatin in response to KLF4 may interfere with recognition of CArG box DNA by SRF. This is consistent with data in Figures 1 and 2 demonstrating that the presence of nuclease-sensitive, accessible CArG box DNA may be an important requirement for SRF binding. Indeed, the crystal structure of SRF bound to CArG box DNA showed that SRF makes several critical contacts with DNA minor groove phosphates that would be obscured if this DNA were compacted into nucleosomes $(18,36)$. H4Ac has been shown to relieve chromatin compaction by disrupting contacts between $\mathrm{H} 4$ tails and $\mathrm{H} 2 \mathrm{~A}$ acidic patch domains of adjacent nucleosomes, disrupting histone-DNA contacts and recruitment of ATP-dependent chromatin remodeling complexes (18-21). H4Ac may operate at SMC genes by any of these mechanisms.
Collectively, the data presented in Figures 3 and 4 suggest a model whereby the SMC-specific presence of H4Ac and $\mathrm{H} 3 \mathrm{~K} 4 \mathrm{dMe}$ at SMC CArG boxes may support a chromatin environment that is permissive for binding of myocardin/SRF complexes. Figure 8 depicts this model, wherein H4Ac facilitates a "relaxed" chromatin structure that provides accessible CArG box DNA for recognition by SRF and H3K4dMe further facilitates binding of myocardin/SRF ternary complexes by providing a docking site for myocardin (or myocardin-associated factors). Signaling pathways that target either KLF4/H4Ac or myocardin expression could thus be utilized by environmental cues to regulate SMC gene expression. Indeed, both PDGF-BB treatment of cultured SMCs and balloon-catheter injury to rat vasculature can increase expression of KLF4 and decrease expression of myocardin $(10,13)$, accompanied by reversible loss of H4Ac and SRF binding to SMC gene CArG box chromatin (Figure 7). Collectively, these results suggest that the balance between myocardin and KLF4 may play an important regulatory role during repression of SMC gene expression under pathophysiological conditions, in part through epigenetic control of SRF binding to CArG box chromatin. This suggests a novel mechanism whereby epigenetic control of chromatin structure may play an important role in regulating SMC gene expression in response to environmental cues that influence SMC differentiation during both normal development and vascular disease states (Figure 8).

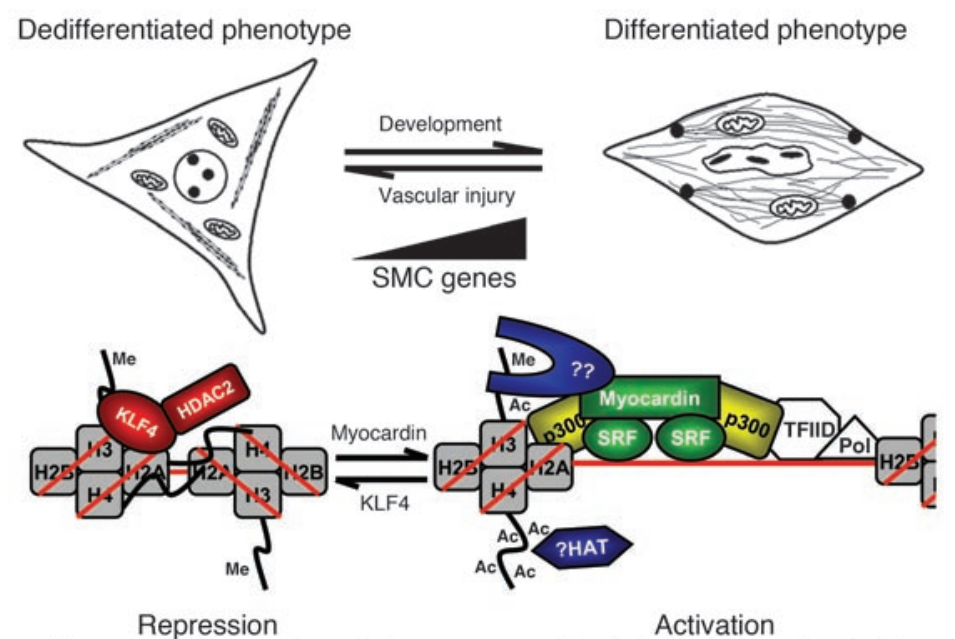

("compacted" CArG promoter region)

("permissive" CArG promoter region)

\section{Figure 8}

Model for epigenetic regulation of SRF binding to CArG box chromatin. Gray squares represent histone octamers with red DNA strands wrapped around them. The dark lines with Me (methyl groups) and Ac (acetyl groups) protruding from $\mathrm{H} 3$ and $\mathrm{H} 4$ represent histone tail domains that are subject to $\mathrm{H} 4$ and $\mathrm{H} 3$ acetylation and H3 Lys4 methylation. In this model, signals such as vascular injury that repress myocardin and/or recruit KLF4-dependent HDAC activity at SMC gene promoters result in loss of SRF binding and transcriptional repression of these genes, to promote the dedifferentiated phenotype. In contrast, in the absence of KLF4, SRF is able to recognize accessible CArG box sequences within "open" chromatin containing $\mathrm{H} 4 \mathrm{Ac}$, synergizing with docking of myocardin to H3K4dMe, to facilitate SRF binding to chromatin and transcriptional activation, which promotes SMC differentiation. The blue protein labeled "??" represents a putative myocardin-accessory factor that may assist myocardin in docking to methylated histones near CArG DNA sequences, to help tether and/or stabilize SRF binding to SMC gene chromatin, which is enriched with $\mathrm{H} 3 \mathrm{~K} 4 \mathrm{dMe}$ in SMCs. 


\section{Methods}

Cell culture, virus infection, and stable transfections. Rat aortic SMCs were cultured in $10 \%$ serum. Rat aortic ECs were a generous gift of Joel Linden (University of Virginia), and cultured in $15 \%$ serum supplemented with endothelial mitogen and heparin. L6 myoblasts were purchased from ATCC and cultured in $2 \%$ serum supplemented with sodium pyruvate for 7 days to induce myotube formation. A404 cells were cultured with or without retinoic acid (which induces expression of all known SMC genes) as described previously (11). Mouse ES cells were cultured on plastic dishes in murine embryonic fibroblast-conditioned media to maintain them in an undifferentiated state. Mouse SMCs were isolated from embryoid bodies (37) and cultured in $20 \%$ serum.

For adenovirus experiments, rat aortic SMCs were infected for 24 hours at an MOI of 50, and rat aortic ECs were infected at an MOI of 100. These MOI values result in $100 \%$ infection efficiencies for these cells, as determined by infection with GFP-expressing viruses. For tail vein injections, $3 \times 10^{9} \mathrm{PFU}$ of total virus was injected, with equal PFU $\left(1.5 \times 10^{9}\right)$ of CMVmyocardin, CMV-KLF4, or CMV-empty control virus to balance viral load. Myocardin and KLF4 adenoviral expression vectors have been described previously $(10,13)$. We also constructed an adenovirus harboring a previously described siRNA to myocardin (38), driven by a minimal H1 promoter. SMCs were infected for 48 hours at an MOI of 100 during siRNA experiments. Details of the cloning procedure are available in ref. 38 .

For stable transfection of rat aortic SMCs, the integrase system was employed (39). Integrase results in selective insertion of transgenes into approximately 7 non-heterochromatic intergenic chromosomal locations containing so-called attP sites (40), by use of a $\phi$-integrase that integrates $a t t B$ sites present in the sequence of transfected plasmids into genomic att $P$ sites. This system has been shown to recapitulate expression patterns of endogenous genes (39). We cloned the full-length wild-type (containing a 2-bp substitution to allow discrimination from the endogenous promoter) and triple CArG mutant $\alpha$-SMA promoter-enhancer driving expression of luciferase into plasmids containing attB sites flanking the cloning site and a SV40 promoter driving a zeocin resistance gene in the opposite orientation. These plasmids were transfected with FuGENE reagent (Roche Diagnostics Corp.) at an integrase/plasmid ratio of 15:1 into cultured rat aortic SMCs, and colonies representing clonal cell lines were selected with zeocin treatment. We isolated multiple clones from 2 independently derived rat aortic SMC lines and measured luciferase activity. All clones carrying wild-type promoter-enhancers displayed high luciferase activity, whereas all clones carrying CArG mutant promoter-enhancers displayed very low to absent luciferase activity compared with wild type (data not shown). Two clones for each construct were analyzed by ChIP, and results from a representative sample are reported.

ChIP, protein immunoprecipitation, and Western blotting. ChIP was performed on cultured cells and whole blood as described previously $(10,41)$. For in vivo ChIP of rat aortas and mouse liver, animals were euthanized and organs quickly dissected out from surrounding tissue, washed in ice-cold PBS to remove blood and debris, snap frozen in liquid nitrogen, and stored at $-80^{\circ} \mathrm{C}$. Adventitia was stripped from aortas during the PBS wash. The organs were later crushed in liquid nitrogen with mortar and pestle on dry ice, transferred directly to $37^{\circ} \mathrm{C} 1 \%$ formaldehyde for 10 minutes, washed 4 times with ice-cold PBS, and taken through the rest of the ChIP procedure with an extra spin included upon addition of SDS lysis buffer, followed by removal of the supernatant from the pelleted extracellular debris. Nuclease digestion experiments were performed as ChIP experiments, except that chromatin was digested to mono- and dinucleosomes with micrococcal nuclease rather than sonicated, and there was no incubation with antibody. DNA extracted from ECs amplified more efficiently at $\alpha$-SMA and $S M-M H C$ than DNA extracted from SMCs, indicating that this DNA was not digested as efficiently by nuclease digestion of chromatin in ECs as in SMCs and was therefore less accessible to nuclease digestion at ECs (Figure 1B). All ChIP data are representative of $2-3$ independent experiments, with each experiment performed in duplicate. Real-time PCR primers and conditions for ChIP and RT-PCR are provided in the supplemental materials. Immunoprecipitations and Western blotting were performed according to standard protocols (42). All antibodies were from Upstate USA Inc., except rabbit anti-SRF, TFIID, and Elk-1 (Santa Cruz Biotechnology Inc.), anti-HDAC2 (Zymed Laboratories Inc.), and anti-FLAG (Sigma-Aldrich). For peptide binding assays (22), $200 \mu \mathrm{g}$ of precleared SMC nuclear extract was incubated with $2-10 \mu$ g of biotinylated peptide (Upstate USA Inc.) in binding buffer ( $150 \mathrm{mM} \mathrm{NaCl}, 50 \mathrm{mM}$ HEPES, $0.1 \%$ Tween, $10 \%$ glycerol, protease inhibitors) for 2 hours, and $10 \mu \mathrm{g}$ mouse FLAG antisera was used to immunoprecipitate FLAG-myocardin overnight at $4^{\circ} \mathrm{C}$ from the extracts. Immunoprecipitates were collected with protein $\mathrm{G}$ beads, washed 2 times with binding buffer, run on $15 \%$ SDS-PAGE gels, and subjected to Western blotting with HRP-conjugated streptavidin (Upstate USA Inc.). An amount of supernatant corresponding to one-twentieth the amount of immunoprecipitate was run in parallel as a loading control.

Assessment of mouse transgene expression. Transgenic mice expressing an $S M-M H C$ promoter-reporter ( $\beta$-gal) construct have been previously described (6). Transgenic mice were anesthetized, and purified viruses were injected intravenously via the tail vein in injectable $0.9 \% \mathrm{NaCl}$. After 7 days, mice were euthanized and perfused with PBS, and a section of the liver was removed for RNA and ChIP processing. Mice were then perfusion fixed with $2 \%$ formaldehyde $/ 0.2 \%$ glutaraldehyde and tissues harvested and stained for $\beta$-gal activity using X-gal (5-bromo-4-chloro3 -indolyl-bD-galactoside; Sigma-Aldrich). Care was taken to ensure that all samples were stained under the same conditions in parallel on the same day. The aorta was processed for routine histology, and sections were counterstained with eosin. All animal experiments were approved by the Animal Care and Use Committee Institutional Review Board at the University of Virginia Health Sciences Center.

Rat aorta and carotid balloon injury model. Male Sprague-Dawley rats (350-400 g; Zivic Laboratories Inc.) were anesthetized, and acute injury to the thoracic aorta and left common carotid artery was made with a $2 \mathrm{~F}$ Fogarty balloon catheter as described previously (33). At the indicated times after injury (Figure 7D), animals were euthanized, and the injured thoracic aorta and injured left carotid were removed and processed accordingly for ChIP or mRNA analyses. The uninjured right carotid was used as the control for the injured left carotid, and sham-operated rats were used for the thoracic aorta control. Injury experiments were replicated in our laboratory and by Zivic Laboratories Inc., and data from representative samples are reported.

Statistics. Data were analyzed for statistical significance using the 2-tailed Student's $t$ test (SigmaStat 3.1; Systat Software Inc.). Error bars represent SEM. $P$ values less than 0.05 were considered statistically significant.

\section{Acknowledgments}

The authors would like to thank Rupa Tripathe, Diane Raines, Mary McCanna, and Zaiming Luo for expert technical assistance and members of the Owens laboratory for helpful discussions.

Received for publication August 5, 2005, and accepted in revised form October 25, 2005.

Address correspondence to: Gary K. Owens, Department of Molecular Physiology and Biological Physics, University of Virginia Health Sciences Center, 415 Lane Road MR5, Room 1226, Charlottesville, Virginia 22908, USA. Phone: (434) 924-5993; Fax: (434) 982-0055; E-mail: gko@virginia.edu. 
1. Owens, G.K., Kumar, M.S., and Wamhoff, B.R. 2004. Molecular regulation of vascular smooth muscle cell differentiation in development and disease. Physiol. Rev. 84:767-801.

2. Hoofnagle, M.H., Wamhoff, B.R., and Owens, G.K 2004. Lost in transdifferentiation. J. Clin. Invest. 113:1249-1251. doi:10.1172/JCI200421761.

3. Hu, Y., et al. 2004. Abundant progenitor cells in the adventitia contribute to atherosclerosis of vein grafts in ApoE-deficient mice. J. Clin. Invest. 113:1258-1265. doi:10.1172/JCI200419628.

4. Sata, M., et al. 2002. Hematopoietic stem cells differentiate into vascular cells that participate in the pathogenesis of atherosclerosis. Nat. Med. 8:403-409.

5. Glaser, R., Lu, M.M., Narula, N., and Epstein, J.A. 2002. Smooth muscle cells, but not myocytes, of host origin in transplanted human hearts. Circulation. 106:17-19.

6. Regan, C.P., Adam, P.J., Madsen, C.S., and Owens, G.K. 2000. Molecular mechanisms of decreased smooth muscle differentiation marker expression after vascular injury. J. Clin. Invest. 106:1139-1147.

7. Miano, J.M. 2003. Serum response factor: toggling between disparate programs of gene expression. J. Mol. Cell. Cardiol. 35:577-593.

8. Dandre, F., and Owens, G.K. 2004. Platelet-derived growth factor-BB and Ets-1 transcription factor negatively regulate transcription of multiple smooth muscle cell differentiation marker genes. Am. J. Physiol. Heart Circ. Physiol. 286:H2042-H2051.

9. Wang, Z., et al. 2004. Myocardin and ternary complex factors compete for SRF to control smooth muscle gene expression. Nature. 428:185-189.

10. Hendrix, J.A., et al. 2005. 5' CArG degeneracy in smooth muscle $\alpha$-actin is required for injuryinduced gene suppression in vivo. J. Clin. Invest. 115:418-427. doi:10.1172/JCI200522648.

11. Manabe, I., and Owens, G.K. 2001. Recruitment of serum response factor and hyperacetylation of histones at smooth muscle-specific regulatory regions during differentiation of a novel P19-derived in vitro smooth muscle differentiation system. Circ. Res. 88:1127-1134.

12. Qiu, P., and Li, L. 2002. Histone acetylation and recruitment of serum responsive factor and CREBbinding protein onto SM22 promoter during SM22 gene expression. Circ. Res. 90:858-865.

13. Liu, Y., et al. 2004. Kruppel-like factor 4 abrogates myocardin-induced activation of smooth muscle gene expression. J. Biol. Chem. 280:9719-9727.

14. Wamhoff, B.R., et al. 2004. A G/C element mediates repression of the SM22alpha promoter within phenotypically modulated smooth muscle cells in experimental atherosclerosis. Circ. Res. 95:981-988.
15. Wamhoff, B.R., et al. 2004. L-type voltage-gated $\mathrm{Ca} 2+$ channels modulate expression of smooth muscle differentiation marker genes via a Rho kinase/myocardin/SRF-dependent mechanism. Circ. Res. 95:406-414.

16. Spotswood, H.T., and Turner, B.M. 2002. An increasingly complex code. J. Clin. Invest. 110:577-582. doi:10.1172/JCI200216547.

17. Jenuwein, T., and Allis, C.D. 2001. Translating the histone code. Science. 293:1074-1080.

18. Luger, K., Mader, A.W., Richmond, R.K., Sargent, D.F., and Richmond, T.J. 1997. Crystal structure of the nucleosome core particle at 2.8[thinsp]A resolution. Nature. 389:251-260.

19. Dorigo, B., Schalch, T., Bystricky, K., and Richmond, T.J. 2003. Chromatin fiber folding: requirement for the histone $\mathrm{H} 4 \mathrm{~N}$-terminal tail. J. Mol. Biol. 327:85-96.

20. Fan, J.Y., Rangasamy, D., Luger, K., and Tremethick, D.J. 2004. H2A.Z alters the nucleosome surface to promote HP1alpha-mediated chromatin fiber folding. Mol. Cell. 16:655-661.

21. Narlikar, G.J., Fan, H.Y., and Kingston, R.E. 2002. Cooperation between complexes that regulate chromatin structure and transcription. Cell. 108:475-487.

22. Pray-Grant, M.G., Daniel, J.A., Schieltz, D., Yates, J.R., and Grant, P.A. 2005. Chd1 chromodomain links histone $\mathrm{H} 3$ methylation with SAGA- and SLIK-dependent acetylation. Nature. 433:434-438.

23. Cao, D., et al. 2005. Modulation of smooth muscle gene expression by association of histone acetyltransferases and deacetylases with myocardin. Mol. Cell. Biol. 25:364-376.

24. Mack, C.P., Thompson, M.M., Lawrenz-Smith, S., and Owens, G.K. 2000. Smooth muscle alpha-actin CArG elements coordinate formation of a smooth muscle cell-selective, serum response factor-containing activation complex. Circ. Res. 86:221-232.

25. Schubeler, D., et al. 2004. The histone modification pattern of active genes revealed through genomewide chromatin analysis of a higher eukaryote. Genes Dev. 18:1263-1271.

26. Wang, D., et al. 2001. Activation of cardiac gene expression by myocardin, a transcriptional cofactor for serum response factor. Cell. 105:851-862.

27. Santos-Rosa, H., et al. 2003. Methylation of histone H3 K4 mediates association of the Isw1p ATPase with chromatin. Mol. Cell. 12:1325-1332.

28. Wysocka, J., et al. 2005. WDR5 associates with histone $\mathrm{H} 3$ methylated at $\mathrm{K} 4$ and is essential for H3 K4 methylation and vertebrate development. Cell. 121:859-872.

29. Suzuki, T., Aizawa, K., Matsumura, T., and Nagai, R. 2005. Vascular implications of the Kruppel-like family of transcription factors. Arterioscler. Thromb. Vasc. Biol. 25:1135-1141.

30. Noti, J.D., Johnson, A.K., and Dillon, J.D. 2005. The leukocyte integrin gene CD11d is repressed by gut-enriched Kruppel-like factor 4 in myeloid cells. J. Biol. Chem. 280:3449-3457.

31. Adam, P.J., Regan, C.P., Hautmann, M.B., and Owens, G.K. 2000. Positive- and negative-acting Kruppel-like transcription factors bind a transforming growth factor beta control element required for expression of the smooth muscle cell differentiation marker SM22alpha in vivo. J. Biol. Chem. 275:37798-37806.

32. Liu, Y., Sinha, S., and Owens, G. 2003. A transforming growth factor-beta control element required for SM alpha-actin expression in vivo also partially mediates GKLF-dependent transcriptional repression. J. Biol. Chem. 278:48004-48011.

33. Clowes, A.W., Reidy, M.A., and Clowes, M.M. 1983. Kinetics of cellular proliferation after arterial injury. I. Smooth muscle growth in the absence of endothelium. Lab. Invest. 49:327-333.

34. Kook, H., et al. 2003. Cardiac hypertrophy and histone deacetylase-dependent transcriptional repression mediated by the atypical homeodomain protein Hop. J. Clin. Invest. 112:863-871. doi:10.1172/JCI200319137.

35. Yang, S.H., and Sharrocks, A.D. 2004. SUMO promotes HDAC-mediated transcriptional repression. Mol. Cell. 13:611-617.

36. Pellegrini, L., Tan, S., and Richmond, T.J. 1995. Structure of serum response factor core bound to DNA. Nature. 376:490-498.

37. Sinha, S., Hoofnagle, M.H., Kingston, P.A., McCanna, M.E., and Owens, G.K. 2004. Transforming growth factor-beta1 signaling contributes to development of smooth muscle cells from embryonic stem cells. Am. J. Physiol. Cell Physiol. 287:C1560-C1568.

38. Yoshida, T., Hoofnagle, M.H., and Owens, G.K. 2004. Myocardin and Prx1 contribute to angiotensin II-induced expression of smooth muscle alphaactin. Circ. Res. 94:1075-1082.

39. Thyagarajan, B., Olivares, E.C., Hollis, R.P., Ginsburg, D.S., and Calos, M.P. 2001. Site-specific genomic integration in mammalian cells mediated by phage \{phi\}C31 integrase. Mol. Cell. Biol. 21:3926-3934.

40. Held, P.K., et al. 2005. In vivo correction of murine hereditary tyrosinemia type I by phiC31 integrasemediated gene delivery. Mol. Ther. 11:399-408.

41. Litt, M., Simpson, M., Recillas-Targa, F., Prioleau, M., and Felsenfeld, G. 2001. Transitions in histone acetylation reveal boundaries of three separately regulated neighboring loci. EMBOJ. 20:2224-2235.

42. Upstate USA Inc. 2005. Procedures and protocols. http://www.upstate.com/misc/protocols.q. 